\title{
Segre maps and entanglement for multipartite systems of indistinguishable particles
}

\author{
Janusz Grabowski* \\ Faculty of Mathematics and Natural Sciences, College of Sciences \\ Cardinal Stefan Wyszyński University, \\ Wóycickiego 1/3, 01-938 Warszawa, Poland \\ Marek Kuśt \\ Center for Theoretical Physics, Polish Academy of Sciences, \\ Aleja Lotników 32/46, 02-668 Warszawa, Poland \\ Giuseppe Marmo \\ Dipartimento di Scienze Fisiche, Università "Federico II" di Napoli \\ and Istituto Nazionale di Fisica Nucleare, Sezione di Napoli, \\ Complesso Universitario di Monte Sant Angelo, \\ Via Cintia, I-80126 Napoli, Italy
}

April 11, 2018

\begin{abstract}
We elaborate the concept of entanglement for multipartite system with bosonic and fermionic constituents and its generalization to systems with arbitrary parastatistics. The entanglement is characterized in terms of generalized Segre maps, supplementing thus an algebraic approach to the problem by a more geometric point of view.
\end{abstract}

Key words: entanglement, tensor product, symmetry group, Bose statistics, Fermi statistics, parastatistics, Young diagram, Segre map.

PACS: 03.65.Aa, 03.67.Mn, 02.10.Xm.

MSC 2000: 81P40, 81P16 (Primary); 15A69, 81R05 (Secondary).

\section{Introduction}

The possibility of identifying subsystems states in a given total state of a composite quantum system goes under the name of separability. In the case of pure states such a possibility is guaranteed if the composite state takes the form of the tensor product of subsystems states.

On the other hand, with the advent of Quantum Field Theory, we have identified elementary particles which are either bosons or fermions. As a matter of fact, according to the spin-statistics theorem all particles are either bosons or fermions. The difference is that a state is unchanged by the interchange of two identical bosons, whereas it changes the sign under the interchange of two identical fermions. The characterization of fermionic states contains already the lack of the factorization of the total state of the

\footnotetext{
*email: jagrab@impan.pl

$\dagger$ email: marek.kus@cft.edu.pl

‡email: marmo@na.infn.it
} 
composite system. According to usual wisdom, this would always imply the presence of an entanglement. In our opinion this state of affairs cannot be maintained, so there is a need of a refinement of the notion of entanglement that describes better the situation when we are dealing with bosons and fermions or even with 'parabosons' or 'parafermions' arising from potentially meaningful parastatistics [1, 2].

In [3] we analyzed a concept of entanglement for a multipartite system with bosonic and fermionic constituents in purely algebraic way using the the representation theory of the underlying symmetry groups. Correlation properties of indistinguishable particles become relevant when subsystems are no longer separated by macroscopic distances, like e.g. in quantum gates based on quantum dots, where they are confined to the same spatial regions 4 . In our approach to bosons and fermions we adopted the concept of entanglement put forward in [4, 5] for fermionic systems and extended in [6, 7] in a natural way to bosonic ones. 1 .

The problem of quantifying and measuring entanglement in systems of many indistinguishable particles remains a topic of vivid investigations from different points of view. Like in the case of distinguishable particles, also nonclassical correlations of indistinguishable particles can be studied with the use of various characteristics, e.g., a suitably adapted concept of the entropy of a state [14, [15] or by mapping the Fock spaces onto spaces of qubits [16, 17, elaborating thus a concept of the so called mode entanglement proposed earlier by Zanardi [18, [19. Another area of the current theoretical research on indistinguishable particles concentrates around problems of identifying and measuring the entanglement in realistic experimental circumstances [20, [21. An exhaustive exposition of these aspects of the theory, out of the scope of the present paper, is contained in a recent review article by Tichy, Mintert, and Buchleitner [22].

Our approach appeared to be sufficiently general to define entanglement also for systems with an arbitrary parastatistics in a consistent and unified way. For pure states we defined the S-rank, generalizing the notion of the Schmidt rank for distinguishable particles and playing an analogous role in the characterization of the degree of entanglement among particles with arbitrary exchange symmetry (parastatistics).

In the algebraic geometry, a canonical embedding of the product $\mathbb{C} P^{n-1} \times \mathbb{C} P^{m-1}$ of complex projective spaces into $\mathbb{C} P^{n m-1}$ is known under the name the Segre embedding (or the Segre map). In the quantum mechanical context, the complex projective space $\mathbb{C} P^{n-1}$ represents pure states in the Hilbert space $\mathbb{C}^{n}$, and $\mathbb{C} P^{n m-1}$ represent pure states in $\mathbb{C}^{n} \otimes \mathbb{C}^{m}$, so that the Segre embedding gives us a geometrical description of separable pure states and, as shown in [8, 9, this description can be extended also to mixed states.

In the present paper we give a geometric description of the entanglement for systems with arbitrary symmetry (with respect to exchanging of subsystems) in terms of generalized Segre embeddings associated with particular parastatistics. This description is complementary to the one presented in 3 ] in terms of the S-rank. For systems with arbitrary exchange symmetries, unlike for the systems of distinguishable particles, the spaces of states are not, in general, projectivizations of the full tensor products of the underlying Hilbert spaces of subsystems, but rather some parts of them. We show in the following how to extend properly the concept of the Segre embedding to achieve a geometric description analogous to that for distinguishable particles. This approach uses a unifying mathematical framework based on the representation theory and strongly suggesting certain concepts of the separability, thus of the entanglement, in the case of indistinguishable particles. For physicists, this approach may be viewed as being too mathematical and abstract, but in our opinion it covers exactly the logic structure of the notion of entanglement for systems of particles with some symmetries.

In the next section we shortly review the relevant concepts of composite systems of distinguishable particles, their description in terms of the classical Segre maps, as well as entanglement measures for systems of distinguishable particles. In sections 3 and 4 we give a brief review of the algebraic description of the entanglement for bosons and fermions in terms of the S-rank of tensors presented in [3]. The main results are contained in Sections 5-7 where we construct the Segre maps, first for bosons and fermions and, finally, for systems with an arbitrary parastatistics. The constructions are based on Theorem 6.1 which relates simple tensor of a given parastatistics with the corresponding Young diagram.

\footnotetext{
${ }^{1}$ For discussion of a slightly different treatment of bosons in [10, 11, 12, 13] see the Introduction and Conclusion sections of $[3]$.
} 


\section{Composite systems, separability, and entanglement}

Let $\mathcal{H}$ be a Hilbert space with a Hermitian product $\langle\cdot \mid \cdot\rangle, g l(\mathcal{H})$ be the vector space of complex linear operators on $\mathcal{H}, G L(\mathcal{H})$ be the group of invertible operators from $g l(\mathcal{H})$, and $U(\mathcal{H})$ be its subgroup of unitary operators on $\mathcal{H}$. For simplicity, we will assume that $\mathcal{H}$ is finite-dimensional, $\operatorname{say} \operatorname{dim}(\mathcal{H})=n$, but a major part of our work remains valid also for Hilbert spaces of infinite dimensions. Note only that in the infinite dimensions the corresponding tensor product $\mathcal{H}_{1} \otimes \mathcal{H}_{2}$ is the tensor product in the category of Hilbert spaces, i.e., corresponding to the Hilbert-Schmidt norm.

With $u(\mathcal{H})$ we will denote the Lie algebra of the Lie group $U(\mathcal{H})$ consisting of anti-Hermitian operators, while $u^{*}(\mathcal{H})$ will denote its dual interpreted as the Euclidean space of Hermitian operators with the scalar product

$$
\langle A, B\rangle_{u^{*}}=\frac{1}{2} \operatorname{Tr}(A B) .
$$

The space of non-negatively defined operators from $g l(\mathcal{H})$, i.e. of those $\rho \in g l(\mathcal{H})$ which can be written in the form $\rho=T^{\dagger} T$ for a certain $T \in g l(\mathcal{H})$, we denote as $\mathcal{P}(\mathcal{H})$. It is a convex cone in $g l(\mathcal{H})$ and the set of density states $\mathcal{D}(\mathcal{H})$ is distinguished in $\mathcal{P}(\mathcal{H})$ by the normalizing condition $\operatorname{Tr}(\rho)=1$. We will regard $\mathcal{P}(\mathcal{H})$ and $\mathcal{D}(\mathcal{H})$ as embedded in $u^{*}(\mathcal{H})$, so that the space $\mathcal{D}(\mathcal{H})$ of density states is a convex set in the affine hyperplane in $u^{*}(\mathcal{H})$ determined by the equation $\operatorname{Tr}(\rho)=1$. As the difference of two Hermitian operators of trace 1 (the vector connection two points in the affine hyperplane) is a Hermitian operator of trace 0 , the model vector spaces of this affine hyperplane is therefore canonically identified with the space of Hermitian operators with the trace 0.

Denote the set of all operators from $\mathcal{D}(\mathcal{H})$ of rank $k$ with $\mathcal{D}^{k}(\mathcal{H})$. It is well known that the set of extreme points of $\mathcal{D}(\mathcal{H})$ coincides with the set $\mathcal{D}^{1}(\mathcal{H})$ of pure states, i.e. the set of one-dimensional orthogonal projectors $\rho_{x}=|x\rangle\langle x|,\|x\|=1$. Hence, every element of $\mathcal{D}(\mathcal{H})$ is a convex combination of points from $\mathcal{D}^{1}(\mathcal{H})$. The space $\mathcal{D}^{1}(\mathcal{H})$ of all pure states can be identified with the complex projective space $\mathbb{P H} \simeq \mathbb{C} P^{n-1}$ via the projection

$$
\mathcal{H} \backslash\{0\} \ni x \mapsto \rho_{x}=\frac{|x\rangle\langle x|}{\|x\|^{2}} \in \mathcal{D}^{1}(\mathcal{H})
$$

which identifies the points of the orbits of the action in $\mathcal{H}$ of the multiplicative group $\mathbb{C} \backslash\{0\}$ by complex homotheties. It is well known that the complex projective space $\mathbb{P} \mathcal{H}=\mathcal{D}^{1}(\mathcal{H})$ is canonically a Kähler manifold. The symplectic structure on $\mathcal{D}^{1}(\mathcal{H}) \subset u^{*}(\mathcal{H})$ is the canonical symplectic structure of an $U(\mathcal{H})$-coadjoint orbit, and the metric, called the Fubini-Study metric is just the metric induced from the embedding of $\mathcal{D}^{1}(\mathcal{H})$ into the Euclidean space $u^{*}(\mathcal{H})$. This is the best known compact Kähler manifold in the algebraic geometry.

Suppose now that our Hilbert space has a fixed decomposition into the tensor product of two Hilbert spaces, $\mathcal{H}=\mathcal{H}^{1} \otimes \mathcal{H}^{2}$. This additional input is crucial in studying composite quantum systems. Observe first that the tensor product map

$$
\otimes: \mathcal{H}^{1} \times \mathcal{H}^{2} \rightarrow \mathcal{H}=\mathcal{H}^{1} \otimes \mathcal{H}^{2}
$$

associates the product of rays with a ray, so it induces a canonical embedding on the level of complex projective spaces,

$$
\begin{aligned}
\text { Seg }: \mathbb{P} \mathcal{H}^{1} \times \mathbb{P} \mathcal{H}^{2} & \rightarrow \mathbb{P} \mathcal{H}=\mathbb{P}\left(\mathcal{H}^{1} \otimes \mathcal{H}^{2}\right), \\
\left(\left|x^{1}\right\rangle\left\langle x^{1}|,| x^{2}\right\rangle\left\langle x^{2}\right|\right) & \mapsto\left|x^{1} \otimes x^{2}\right\rangle\left\langle x^{1} \otimes x^{2}\right|, \quad\left\|x^{1}\right\|=\left\|x^{2}\right\|=1
\end{aligned}
$$

This embedding of the product of complex projective spaces into the projective space of the tensor product is called in the literature the Segre embedding 24]. Note that the elements of the image $\operatorname{Seg}\left(\mathbb{P} \mathcal{H}^{1} \times\right.$ $\left.\mathbb{P} \mathcal{H}^{2}\right)$ in $\mathbb{P H}=\mathbb{P}\left(\mathcal{H}^{1} \otimes \mathcal{H}^{2}\right)$ are usually called separable pure states (separable with respect to the decomposition $\left.\mathcal{H}=\mathcal{H}^{1} \otimes \mathcal{H}^{2}\right)$.

The Segre embedding is related to the (external) tensor product of the basic representations of the unitary groups $U\left(\mathcal{H}^{1}\right)$ and $U\left(\mathcal{H}^{2}\right)$, i.e. with the representation of the direct product group in $\mathcal{H}=\mathcal{H}^{1} \otimes \mathcal{H}^{2}$,

$$
\begin{aligned}
U\left(\mathcal{H}^{1}\right) \times U\left(\mathcal{H}^{2}\right) \ni\left(T_{1}, T_{2}\right) & \mapsto T_{1} \otimes T_{2} \in U(\mathcal{H})=U\left(\mathcal{H}^{1} \otimes \mathcal{H}^{2}\right) \\
\left(T_{1} \otimes T_{2}\right)\left(x^{1} \otimes x^{2}\right) & =T_{1}\left(x^{1}\right) \otimes T_{2}\left(x^{2}\right) .
\end{aligned}
$$


Note that $T_{1} \otimes T_{2}$ is unitary, since the Hermitian product in $\mathcal{H}$ is related to the Hermitian products in $\mathcal{H}^{1}$ and $\mathcal{H}^{2}$ by

$$
\left\langle x^{1} \otimes x^{2}, y^{1} \otimes y^{2}\right\rangle_{\mathcal{H}}=\left\langle x^{1}, y^{1}\right\rangle_{\mathcal{H}^{1}} \cdot\left\langle x^{2}, y^{2}\right\rangle_{\mathcal{H}^{2}}
$$

The above group embedding gives rise to the corresponding embedding of Lie algebras or, by our identification, of their duals which, with some abuse of notation, we will denote by

$$
\operatorname{Seg}: u^{*}\left(\mathcal{H}^{1}\right) \times u^{*}\left(\mathcal{H}^{2}\right) \rightarrow u^{*}(\mathcal{H}), \quad(A, B) \mapsto A \otimes B
$$

The original Segre embedding is just the latter map reduced to pure states. In fact, a stronger result holds true [8, 9].

Proposition 2.1. The embedding (7) maps $\mathcal{D}^{k}\left(\mathcal{H}^{1}\right) \times \mathcal{D}^{l}\left(\mathcal{H}^{2}\right)$ into $\mathcal{D}^{k l}(\mathcal{H})$.

Let us denote the image $\operatorname{Seg}\left(\mathcal{D}^{1}\left(\mathcal{H}^{1}\right) \times \mathcal{D}^{1}\left(\mathcal{H}^{2}\right)\right)$, i.e. the set of separable pure states, with $\mathcal{S}^{1}(\mathcal{H})$, and its convex hull, conv $\left(\mathcal{S}^{1}(\mathcal{H})\right)$, i.e. the set of all mixed separable states in $u^{*}(\mathcal{H})$, with $\mathcal{S}(\mathcal{H})$. The states from

$$
\mathcal{E}(\mathcal{H})=\mathcal{D}(\mathcal{H}) \backslash \mathcal{S}(\mathcal{H})
$$

i.e. those which are not separable, are called entangled states. It is well known (see e.g. [8]) that $\mathcal{S}^{1}(\mathcal{H})$ is exactly the set of extremal points of $\mathcal{S}(\mathcal{H})$. What we have just presented is a very simple geometric interpretation of separability and entanglement.

The entangled states play an important role in quantum computing and one of main problems is to decide effectively whether a given composite state is entangled or not. An abstract measurement of entanglement can be based on the following observation (see also [23])

Let $E$ be the set of all extreme points of a compact convex set $K$ in a finite-dimensional real vector space $V$, and let $E_{0}$ be a compact subset of $E$ with the convex hull $K_{0}=\operatorname{conv}\left(E_{0}\right) \subset K$. For every non-negative function $f: E \rightarrow \mathbf{R}_{+}$define its extension (convex roof) $f_{K}: K \rightarrow \mathbf{R}_{+}$by

$$
f_{K}(x)=\inf _{x=\sum t_{i} \alpha_{i}} \sum t_{i} f\left(\alpha_{i}\right)
$$

where the infimum is taken with respect to all expressions of $x$ in the form of convex combinations of points from $E$. Recall that, according to Krein-Milman theorem, $K$ is the convex hull of its extreme points.

Proposition 2.2. For every non-negative continuous function $f: E \rightarrow \mathbf{R}_{+}$which vanishes exactly on $E_{0}$, the function $f_{K}$ is convex on $K$ and vanishes exactly on $K_{0}$

An immediate consequence is the following (cf. [8, 9]).

Corollary 2.1. Let $\mathcal{H}=\mathcal{H}^{1} \otimes \mathcal{H}^{2}$ and let $F: \mathcal{D}^{1}(\mathcal{H}) \rightarrow \mathbf{R}_{+}$be a continuous function which vanishes exactly on on the set $\mathcal{S}^{1}(\mathcal{H})$ of separable pure states. Then,

$$
\mu=F_{\mathcal{D}(\mathcal{H})}: \mathcal{D}(\mathcal{H}) \rightarrow \mathbf{R}_{+}
$$

is a measure of entanglement, i.e. $\mu$ is convex and $\mu(\rho)=0$ if and only if the (mixed) density state $\rho$ is separable. Moreover, if $f$ is taken $U\left(\mathcal{H}^{1}\right) \times U\left(\mathcal{H}^{2}\right)$-invariant, then $\mu$ is $U\left(\mathcal{H}^{1}\right) \times U\left(\mathcal{H}^{2}\right)$-invariant.

Remark 2.1. In the terminology of [23], the convex roof function $F_{\mathcal{D}(\mathcal{H})}$ is entanglement monotone if $F$ is entanglement monotone on pure states.

\section{Tensor algebras, fermions, and bosons}

To describe some properties of systems composed of indistinguishable particles and to fix the notation, let us start with introducing corresponding tensor algebras associated with a Hilbert space $\mathcal{H}$. 
In the tensor power $\mathcal{H}^{\otimes k}=\underbrace{\mathcal{H} \otimes \cdots \otimes \mathcal{H}}_{k-\text { times }}$, we distinguish the subspaces: $\mathcal{H}^{\vee k}=\underbrace{\mathcal{H} \vee \cdots \vee \mathcal{H}}_{k-\text { times }}$ of totally symmetric tensors and $\mathcal{H}^{\wedge k}=\underbrace{\mathcal{H} \wedge \cdots \wedge \mathcal{H}}_{k \text {-times }}$ of totally antisymmetric ones, together with the symmetrization, $\pi_{k}^{\vee}: \mathcal{H}^{\otimes k} \rightarrow \mathcal{H}^{\vee k}$, and antisymmetrization, $\pi_{k}^{\wedge}: \mathcal{H}^{\otimes k} \rightarrow \mathcal{H}^{\wedge k}$, projectors:

$$
\begin{aligned}
& \pi_{k}^{\vee}\left(f_{1} \otimes \cdots \otimes f_{k}\right)=\frac{1}{k !} \sum_{\sigma \in S_{k}} f_{\sigma(1)} \otimes \cdots \otimes f_{\sigma(k)}, \\
& \pi_{k}^{\wedge}\left(f_{1} \otimes \cdots \otimes f_{k}\right)=\frac{1}{k !} \sum_{\sigma \in S_{k}}(-1)^{\sigma} f_{\sigma(1)} \otimes \cdots \otimes f_{\sigma(k)} .
\end{aligned}
$$

Here, $S_{k}$ is the group of all permutations $\sigma:\{1, \ldots, k\} \rightarrow\{1, \ldots, k\}$, and $(-1)^{\sigma}$ denotes the sign of the permutation $\sigma$. Note that with every permutation $\sigma \in S_{k}$ there is a canonically associated unitary operator $U_{\sigma}$ on $\mathcal{H}^{\otimes k}$ defined by

$$
U_{\sigma}\left(f_{1} \otimes \cdots \otimes f_{k}\right)=f_{\sigma(1)} \otimes \cdots \otimes f_{\sigma(k)},
$$

so that the map $\sigma \mapsto U_{\sigma}$ is an injective unitary representation of $S_{k}$ in $\mathcal{H}^{\otimes k}$. We will write simply $\sigma$ instead of $U_{\sigma}$ if no misunderstanding is possible. Symmetric and skew-symmetric tensors are characterized in terms of this unitary action by $\sigma(v)=v$ and $\sigma(v)=(-1)^{\sigma} v$, respectively, for all $\sigma \in S_{k}$

We put, by convention, $\mathcal{H}^{\otimes 0}=\mathcal{H}^{\vee 0}=\mathcal{H}^{\wedge 0}=\mathbb{C}$. It is well known that the obvious structure of a unital graded associative algebra on the graded space $\mathcal{H}^{\otimes}=\otimes_{k=0}^{\infty} \mathcal{H}^{\otimes k}$ (the tensor algebra) induces canonical unital graded associative algebra structures on the spaces $\mathcal{H}^{\vee}=\underset{k=0}{\oplus} \mathcal{H}^{\vee k}$ (called the bosonic Fock space) and $\mathcal{H}^{\wedge}=\bigoplus_{k=0}^{\infty} \mathcal{H}^{\wedge k}$ (called the fermionic Fock space) of symmetric and antisymmetric tensors. This simply means that we have associative multiplications

$$
\begin{gathered}
v_{1} \vee v_{2}=\pi^{\vee}\left(v_{1} \otimes v_{2}\right), \\
w_{1} \wedge w_{2}=\pi^{\wedge}\left(w_{1} \otimes w_{2}\right),
\end{gathered}
$$

where

$$
\pi^{\vee}=\bigoplus_{k=0}^{\infty} \pi_{k}^{\vee}: \mathcal{H}^{\otimes} \rightarrow \mathcal{H}^{\vee}
$$

and

$$
\pi^{\wedge}=\bigoplus_{k=0}^{\infty} \pi_{k}^{\wedge}: \mathcal{H}^{\otimes} \rightarrow \mathcal{H}^{\wedge}
$$

are the symmetrization and antisymmetrization projections. Moreover, these multiplications respect the grading, i.e. $v_{1} \vee v_{2} \in \mathcal{H}^{\vee(k+l)}$ if $v_{1} \in \mathcal{H}^{\vee k}, v_{2} \in \mathcal{H}^{\vee l}$, and $w_{1} \wedge w_{2} \in \mathcal{H}^{\wedge(k+l)}$ if $w_{1} \in \mathcal{H}^{\wedge k}, w_{2} \in \mathcal{H}^{\wedge l}$. Note also that the multiplication in $\mathcal{H}^{\vee}$ is commutative, $v_{1} \vee v_{2}=v_{2} \vee v_{1}$, and the multiplication in $\mathcal{H}^{\wedge}$ is graded commutative, $w_{1} \wedge w_{2}=(-1)^{k_{1} \cdot k_{2}} w_{2} \wedge w_{1}$, for $w_{i} \in \mathcal{H}^{\wedge k_{i}}$.

It is well known that the symmetric tensor algebra $\mathcal{H}^{\vee}$ can be canonically identified with the algebra $\operatorname{Pol}(\mathcal{H})$ of polynomial functions on $\mathcal{H}$. Indeed, any $f \in \mathcal{H}$ can be identified with the linear function $x_{f}$ on $\mathcal{H}$ by means of the Hermitian product: $x_{f}(y)=\langle f \mid y\rangle$. We must stress, however, that the identification $f \mapsto x_{f}$ is anti-linear. This can be extended to an anti-linear isomorphism of commutative algebras in which $f_{1} \vee \cdots \vee f_{k}$ corresponds to the homogenous polynomial $x_{f_{1}} \cdots x_{f_{k}}$. Similarly, one identifies $\mathcal{H}^{\wedge}$ with the Grassmann algebra $\operatorname{Grass}(\mathcal{H})$ of polynomial (super)functions on $\mathcal{H}$. Here, however, with $f \in \mathcal{H}$ we associate a linear function $\xi_{f}$ on $\mathcal{H}$ regarded as and odd function: $\xi_{f} \xi_{f^{\prime}}=-\xi_{f^{\prime}} \xi_{f}$. In the language of supergeometry one speaks about the purely odd vector space $\Pi \mathcal{H}$ obtained from $\mathcal{H}$ by changing the parity.

If we fix a basis $e_{1}, \ldots, e_{n}$ in $\mathcal{H}$ and associate with its elements even linear functions $x_{1}, \ldots, x_{n}$ on $\mathcal{H}$, and odd linear functions $\xi_{1}, \ldots, \xi_{n}$ on $\Pi \mathcal{H}$, then $\mathcal{H}^{\vee}$ can be identified with the algebra of complex polynomials in $n$ commuting variables, $\mathcal{H}^{\vee} \simeq \mathbb{C}\left[x_{1}, \ldots, x_{n}\right]$. Similarly, $\mathcal{H}^{\wedge} \simeq \mathbb{C}\left[\xi_{1}, \ldots, \xi_{n}\right]$, i.e., $\mathcal{H}^{\wedge}$ can be identified with the algebra of complex Grassmann polynomials in $n$ anticommuting variables. The 
subspaces $\mathcal{H}^{\vee k}$ and $\mathcal{H}^{\wedge k}$ correspond to homogenous polynomials of degree $k$. It is straightforward that homogeneous polynomials $x_{1}^{k_{1}} \cdots x_{n}^{k_{n}}$, with $k_{1}+\cdots+k_{n}=k$, form a basis of $\mathcal{H}^{\vee k}$, while homogeneous Grassmann polynomials $\xi_{i_{1}} \wedge \cdots \wedge \xi_{i_{k}}$, with $1 \leq i_{1}<i_{2}<\cdots<i_{k} \leq n$, form a basis of $\mathcal{H}^{\wedge k}$. In consequence, $\operatorname{dim} \mathcal{H}^{\vee k}=\left(\begin{array}{c}n+k-1 \\ k\end{array}\right)$ and $\operatorname{dim} \mathcal{H}^{\wedge k}=\left(\begin{array}{l}n \\ k\end{array}\right)$, so the gradation in the fermionic Fock space is finite-dimensional (for a finite-dimensional $\mathcal{H}$ ).

Note that any basis $\left\{e_{1}, \ldots, e_{n}\right\}$ in $\mathcal{H}$ induces a basis $\left\{e_{i_{1}} \otimes e_{i_{2}} \otimes \cdots \otimes e_{i_{k}} \mid i_{1}, \ldots, i_{k} \in\{1, \ldots, n\}\right\}$ in $\mathcal{H}^{\otimes k}$. Therefore, any $u \in \mathcal{H}^{\otimes k}$ can be uniquely written as a linear combination

$$
u=\sum_{i_{1}, \ldots, i_{k}=1}^{n} u^{i_{1} \ldots i_{k}} e_{i_{1}} \otimes \ldots \otimes e_{i_{k}} .
$$

If $u \in \mathcal{H}^{\vee k}$, then the tensor coefficients $u^{i_{1} \ldots i_{k}}$ are totally symmetric and, after applying the symmetrization projection to (15), we get

$$
u=\sum_{i_{1}, \ldots, i_{k}=1}^{n} u^{i_{1} \ldots i_{k}} e_{i_{1}} \vee \ldots \vee e_{i_{k}}
$$

Similarly, if $u \in \mathcal{H}^{\wedge k}$, the tensor coefficients $u^{i_{1} \ldots i_{k}}$ are totally antisymmetric and

$$
u=\sum_{i_{1}, \ldots, i_{k}=1}^{n} u^{i_{1} \ldots i_{k}} e_{i_{1}} \wedge \ldots \wedge e_{i_{k}}
$$

We will refer to the coefficients $u^{i_{1} \ldots i_{k}}$ as to the coefficients of $u$ in the basis $\left\{e_{1}, \ldots, e_{n}\right\}$.

The Hermitian product in $\mathcal{H}$ has an obvious extension to a Hermitian product in $\mathcal{H}^{\otimes k}$,

$$
\left\langle f_{1} \otimes \cdots \otimes f_{k} \mid g_{1} \otimes \cdots \otimes g_{k}\right\rangle=\prod_{i=1}^{k}\left\langle f_{i} \mid g_{i}\right\rangle
$$

and viewing symmetric and antisymmetric tensors as canonically embedded in the tensor algebra, we find the corresponding Hermitian products in $\mathcal{H}^{\vee k}$ and $\mathcal{H}^{\wedge k}$.

For $f_{1}, \ldots, f_{k} \in \mathcal{H}$ and $g_{1}, \ldots, g_{k} \in \mathcal{H}$, we get

$$
\left\langle f_{1} \vee \cdots \vee f_{k} \mid g_{1} \vee \cdots \vee g_{k}\right\rangle=\frac{1}{(k !)^{2}} \sum_{\sigma, \tau \in S_{k}} \prod_{i=1}^{k}\left\langle f_{\sigma(i)} \mid g_{\tau(i)}\right\rangle=\frac{1}{k !} \operatorname{per}\left(\left\langle f_{i} \mid g_{j}\right\rangle\right)
$$

Here, $\frac{1}{k !} \sum_{\tau \in S_{k}} \prod_{i=1}^{k} a_{i \tau(i)}=\operatorname{per}\left(a_{i j}\right)$ is the permanent of the matrix $A=\left(a_{i j}\right)$. Similarly,

$$
\left\langle f_{1} \wedge \cdots \wedge f_{k} \mid g_{1} \wedge \cdots \wedge g_{k}\right\rangle=\frac{1}{k !} \operatorname{det}\left(\left\langle f_{i} \mid g_{j}\right\rangle\right)
$$

These Hermitian products can be generalized to certain 'pairings' (contractions or inner products) between $\mathcal{H}^{\vee k}$ and $\mathcal{H}^{\vee l}$ on one hand, and $\mathcal{H}^{\wedge k}$ and $\mathcal{H}^{\wedge l}$ on the other, $l \leq k$. For the standard simple tensors $f=f_{1} \otimes \cdots \otimes f_{k} \in \mathcal{H}^{\otimes k}$ and $g=g_{1} \otimes \cdots \otimes g_{l} \in \mathcal{H}^{\otimes l}$, we just put

$$
\imath_{g} f=\left\langle f_{1} \otimes \cdots \otimes f_{l} \mid g_{1} \otimes \cdots \otimes g_{l}\right\rangle f_{l+1} \otimes \cdots \otimes f_{k}
$$

and extend it by linearity to all tensors. It is easy to see now that, if $v=f_{1} \vee \cdots \vee f_{k} \in \mathcal{H}^{\vee k} \subset \mathcal{H}^{\otimes k}$ and $\nu=g_{1} \vee \cdots \vee g_{l} \in \mathcal{H}^{\vee l} \subset \mathcal{H}^{\otimes l}$, then $\imath_{\nu} v \in \mathcal{H}^{\vee(k-l)}$.

Similarly, $\imath_{\omega} w \in \mathcal{H}^{\wedge(k-l)}$, if $w \in \mathcal{H}^{\wedge k} \subset \mathcal{H}^{\otimes k}$ and $\omega \in \mathcal{H}^{\wedge l} \subset \mathcal{H}^{\otimes l}$. Explicitly,

$$
\begin{aligned}
\imath_{g_{1} \vee \cdots \vee g_{l}} f_{1} \vee & \cdots \vee f_{k}=\frac{1}{k ! l !} \sum_{\substack{\sigma \in S_{k} \\
\tau \in S_{l}}} \prod_{j=1}^{l}\left\langle f_{\sigma(j)} \mid g_{\tau(j)}\right\rangle f_{\sigma(l+1)} \otimes \cdots \otimes f_{\sigma(k)} \\
& =\frac{(k-l) !}{k !} \sum_{\substack{S \in S(l, k-l) \\
\tau \in S_{l}}} \prod_{j=1}^{l}\left\langle f_{S(j)} \mid g_{\tau(j)}\right\rangle f_{S(l+1)} \vee \cdots \vee f_{S(k)},
\end{aligned}
$$


where $S(l, k-l)$ denotes the group of all $(l, k-l)$ shuffles. Recall that a permutation $\tau$ in $S_{p+q}$ is a $(p, q)$ shuffle if $\tau(1)<\cdots<\tau(p)$ and $\tau(p+1)<\cdots<\tau(p+q)$.

For skew-symmetric tensors,

$$
\begin{aligned}
\imath_{g_{1} \wedge \cdots \wedge g_{l}} f_{1} \wedge & \cdots \wedge f_{k}=\frac{1}{k ! l !} \sum_{\substack{\sigma \in S_{k} \\
\tau \in S_{l}}}(-1)^{\sigma}(-1)^{\tau} \prod_{j=1}^{l}\left\langle f_{\sigma(j)} \mid g_{\tau(j)}\right\rangle f_{\sigma(l+1)} \otimes \cdots \otimes f_{\sigma(k)} \\
= & \frac{(k-l) !}{k !} \sum_{\substack{S \in S(l, k-l) \\
\tau \in S_{l}}}(-1)^{\sigma}(-1)^{\tau} \prod_{j=1}^{l}\left\langle f_{S(j)} \mid g_{\tau(j)}\right\rangle f_{S(l+1)} \wedge \cdots \wedge f_{S(k)} .
\end{aligned}
$$

In particular,

$$
\imath_{g_{1} \vee \cdots \vee g_{k}} f_{1} \vee \cdots \vee f_{k}=\left\langle f_{1} \vee \cdots \vee f_{k} \mid g_{1} \vee \cdots \vee g_{k}\right\rangle
$$

and

$$
\imath_{g_{1} \wedge \cdots \wedge g_{k}} f_{1} \wedge \cdots \wedge f_{k}=\left\langle f_{1} \wedge \cdots \wedge f_{k} \mid g_{1} \wedge \cdots \wedge g_{k}\right\rangle
$$

Moreover,

$$
\imath_{g_{1} \vee \cdots \vee g_{k-1}} f_{1} \vee \cdots \vee f_{k}=\frac{1}{k !} \sum_{j=1}^{k}\left\langle f_{1} \vee \stackrel{j}{\vee} \vee \vee f_{k} \mid g_{1} \vee \cdots \vee g_{k-1}\right\rangle f_{j}
$$

and

$$
\imath_{g_{1} \wedge \cdots \wedge g_{k-1}} f_{1} \wedge \cdots \wedge f_{k}=\frac{1}{k !} \sum_{j=1}^{k}(-1)^{k-j}\left\langle f_{1} \wedge \stackrel{j}{\vee} \cdot \wedge f_{k} \mid g_{1} \wedge \cdots \wedge g_{k-1}\right\rangle f_{j},
$$

where $\stackrel{j}{v}$ stands for the omission.

\section{The S-rank and entanglement for multipartite Bose and Fermi systems}

There are many concepts of a rank of a tensor used in describing its complexity. One of the simplest and most natural is the one based on the inner product operators defined in the previous section. This rank, called in [3] the $S$-rank and used there to define the entanglement for systems of indistinguishable particles, is a natural generalization of the Schmidt rank of 2-tensors.

Definition 4.1. Let $u \in \mathcal{H}^{\otimes k}$. By the $S$-rank of $u$, we understand the maximum of dimensions of the linear spaces $\imath_{\mathcal{H}}^{k-1} \sigma(u)$, for $\sigma \in S_{k}$, which are the images of the contraction maps

$$
\mathcal{H}^{\otimes(k-1)} \ni \nu \mapsto \imath_{\nu} \sigma(u) \in \mathcal{H} .
$$

Non-zero tensors of minimal S-rank in $\mathcal{H}^{\otimes k}$ (resp., $\mathcal{H}^{\vee k}, \mathcal{H}^{\wedge k}$ ) we will call simple (resp., simple symmetric, simple antisymmetric).

Note that he above definition has its natural counterpart for distinguishable particles, so tensors from $\mathcal{H}_{1} \otimes \cdots \otimes \mathcal{H}_{k}$. We just do the contractions with tensors from $\mathcal{H}_{1} \otimes \cdots \otimes \mathcal{H}_{k-1}$ and the corresponding permutations. If particles are identical, $\mathcal{H}_{i}=\mathcal{H}$, and indistinguishable, e.g. the tensors are symmetric or skew-symmetric, we can skip using permutations. In other words, for $u \in \mathcal{H}^{\vee k}$ (resp., $u \in \mathcal{H}^{\wedge k}$ ), the S-rank of $u$ equals the dimension of the linear space which is the image of the contraction map,

$$
\mathcal{H}^{\vee(k-1)} \ni \nu \mapsto \imath_{\nu} u \in \mathcal{H},
$$

(resp.,

$$
\left.\mathcal{H}^{\wedge(k-1)} \ni \nu \mapsto \imath_{\nu} u \in \mathcal{H}\right) .
$$

Theorem 4.1. ([3]) 
(a) The minimal possible $S$-rank of a non-zero tensor $u \in \mathcal{H}^{\otimes k}$ equals 1 . A tensor $u \in \mathcal{H}^{\otimes k}$ is of S-rank 1 if and only if $u$ is decomposable, i.e., it can be written in the form

$$
u=f_{1} \otimes \cdots \otimes f_{k}, \quad f_{i} \in \mathcal{H}, \quad f_{i} \neq 0 .
$$

Such tensors span $\mathcal{H}^{\otimes k}$.

(b) The minimal possible S-rank of a non-zero tensor $v \in \mathcal{H}^{\vee k}$ equals 1 . A tensor $v \in \mathcal{H}^{\vee k}$ is of $S$-rank 1 if and only if $v$ can be written in the form

$$
v=f \vee \cdots \vee f, \quad f \in \mathcal{H}, \quad f \neq 0 .
$$

Such tensors span $\mathcal{H}^{\vee k}$.

(c) The minimal possible $S$-rank of a non-zero tensor $w \in \mathcal{H}^{\wedge k}$ equals $k$. A tensor $w \in \mathcal{H}^{\wedge k}$ is of S-rank $k$ if and only if $w$ can be written in the form

$$
w=f_{1} \wedge \cdots \wedge f_{k},
$$

where $f_{1}, \ldots, f_{k} \in \mathcal{H}$ are linearly independent. Such tensors span $\mathcal{H}^{\wedge k}$.

In particular, the $S$-rank is 1 for simple and simple symmetric tensors and it is $k$ for simple antisymmetric tensors from $\mathcal{H}^{\wedge k}$. Simple tensors have the form (30), simple symmetric tensors have the form (31), and simple antisymmetric tensors have the form (32).

Using the concept of simple tensors we can define simple (non-entangled or separable) and entangled pure states for multipartite systems of bosons and fermions.

\section{Definition 4.2.}

(a) A pure state $\rho_{x}$ on $\mathcal{H}^{\vee k}$ (resp., on $\mathcal{H}^{\wedge k}$ ), $\rho_{x}=\frac{|x\rangle\langle x|}{\|x\|^{2}}$, with $x \in \mathcal{H}^{\vee k}$ (resp., $x \in \mathcal{H}^{\wedge k}$ ), $x \neq 0$, is called a bosonic (resp., fermionic) simple (or non-entangled) pure state if $x$ is a simple symmetric (resp., antisymmetric) tensor. If $x$ is not simple symmetric (resp., antisymmetric), we call $\rho_{x}$ a bosonic (resp., fermionic) entangled state.

(b) A mixed state $\rho$ on $\mathcal{H}^{\vee k}$ (resp., on $\mathcal{H}^{\wedge k}$ ) we call bosonic (resp., fermionic) simple (or non-entangled) mixed state if it can be written as a convex combination of bosonic (resp., fermionic) simple pure states. In the other case, $\rho$ is called bosonic (resp., fermionic) entangled mixed state.

According to Theorem 4.1, bosonic simple pure $k$-states are of the form

$$
|e \vee \cdots \vee e\rangle\langle e \vee \cdots \vee e|
$$

for unit vectors $e \in \mathcal{H}$, and fermionic simple pure $k$-states are of the form

$$
k !\left|e_{1} \wedge \cdots \wedge e_{k}\right\rangle\left\langle e_{1} \wedge \cdots \wedge e_{k}\right|
$$

for orthonormal systems $e_{1}, \ldots, e_{k}$ in $\mathcal{H}$.

Fixing a base in $\mathcal{H}$ results in defining coefficients $\left[u^{i_{1} \ldots i_{k}}\right]$ of $u \in \mathcal{H}^{\otimes k}$. Formulae characterizing simple tensors, thus simple pure states, can be written in terms of quadratic equations with respect to these coefficients as follows. The corresponding characterization of entangled pure states are obtained by negation of the latter.

Theorem 4.2. ([3])

(a) The pure state $\rho_{u}$, associated with a tensor $u=\left[u^{i_{1} \ldots i_{k}}\right] \in \mathcal{H}^{\otimes k}$, is entangled if and only if there exist $i_{1}, \ldots, i_{k}, j_{1}, \ldots, j_{k}$, and $s=1, \ldots, k$ such that

$$
u^{i_{1} \ldots i_{s} \ldots i_{k}} u^{j_{1} \ldots j_{s} \ldots j_{k}} \neq u^{i_{1} \ldots j_{s} \ldots i_{k}} u^{j_{1} \ldots i_{s} \ldots j_{k}} .
$$


(b) The bosonic pure state $\rho_{v}$, associated with a symmetric tensor $v=\left[v^{i_{1} \ldots i_{k}}\right] \in \mathcal{H}^{\vee k}$, is bosonic entangled if and only if there exist $i_{1}, \ldots, i_{k}, j_{1}, \ldots, j_{k}$, such that

$$
v^{i_{1} \ldots i_{k-1} i_{k}} v^{j_{1} \ldots j_{k-1} j_{k}} \neq v^{i_{1} \ldots i_{k-1} j_{k}} v^{j_{1} \ldots j_{k-1} i_{k}} .
$$

(c) The fermionic pure state $\rho_{w}$, associated with an antisymmetric tensor $w=\left[w^{i_{1} \ldots i_{k}}\right] \in \mathcal{H}^{\wedge k}$, is fermionic entangled if and only if there exist $i_{1}, \ldots, i_{k+1}, j_{1}, \ldots, j_{k-1}$ such that

$$
w^{\left[i_{1} \ldots i_{k}\right.} w^{\left.i_{k+1}\right] j_{1} \ldots j_{k-1}} \neq 0,
$$

where the left-hand side is the antisymmetrization of $w^{i_{1} \ldots i_{k}} w^{i_{k+1} j_{1} \ldots j_{k-1}}$ with respect to the indices $i_{1}, \ldots, i_{k+1}$.

Note that the opposite to (35), $w^{\left[i_{1} \ldots i_{k}\right.} w^{\left.i_{k+1}\right] j_{1} \ldots j_{k-1}}=0$, are sometimes called the Plücker relations.

Example 4.1. Assume that deal with qubit systems, and $|0\rangle,|1\rangle$ is an orthonormal basis in $\mathcal{H}$. The tensor $u=|0\rangle \otimes|0\rangle$ has the S-rank 1:

$$
\imath_{a|0\rangle+b|1\rangle} \sigma(u)=\imath_{a|0\rangle+b|1\rangle}(u)=(a\langle 0|+b\langle 1|) \mid 0\rangle|0\rangle=a|0\rangle
$$

which is the 1-dimensional space spanned by $|0\rangle$, while the tensor $u_{ \pm}=|0\rangle \otimes|1\rangle \pm|1\rangle \otimes|0\rangle$ has the S-rank 2:

$$
\begin{aligned}
\imath_{a|0\rangle+b|1\rangle} \sigma(u)= & \pm \imath_{a|0\rangle+b|1\rangle} u_{ \pm}= \\
& \pm(a\langle 0|+b\langle 1|) \mid 0\rangle|1\rangle \pm(a\langle 0|+b\langle 1|) \mid 1\rangle|0\rangle= \pm a|1\rangle \pm b|0\rangle .
\end{aligned}
$$

Example 4.2. For the GHZ-states $\left|G H Z_{k}\right\rangle$ and W-states $\left|W_{k}\right\rangle$ the S-rank is 2 independently on $k \geq 2$. Indeed, it is clear that contractions of

$$
\left|G H Z_{k}\right\rangle=\frac{1}{\sqrt{2}}\left(|0\rangle^{\otimes k}+|1\rangle^{\otimes k}\right)
$$

with $(k-1)$-tensors give all linear combinations of $|0\rangle$ and $|1\rangle$. The same is true for

$$
\left|W_{k}\right\rangle=\frac{1}{\sqrt{k}}(|0 \cdots 01\rangle+|0 \cdots 10\rangle+\cdots+|1 \cdots 00\rangle)
$$

On the other hand, the S-rank cannot exceed 2 for qubit systems.

Remark 4.1. The S-rank does not distinguish between $\left|G H Z_{k}\right\rangle$ and $\left|W_{k}\right\rangle$. Note however that we can slightly generalize our notion of the S-rank including also contractions $\iota_{\nu} u$ with shorter tensors, i.e. tensors $\nu \in \mathcal{H}^{\otimes(k-l)}$ with $0<l<k$. We have not insist on this generalization in order to avoid additional technical complications. The simple version of the S-rank is sufficient for distinguishing simple tensors. This extended version could be useful in measuring the entanglement.

Example 4.3. If $\left|i_{1}\right\rangle$ and $\left|i_{2}\right\rangle$ are orthonormal sets in $\mathcal{H}_{1}$ and $\mathcal{H}_{2}$, then the S-rank of $u \sum_{i=1}^{r} \lambda_{i}\left|i_{1}\right\rangle \otimes\left|i_{2}\right\rangle$ is $r$, as $\iota_{\mathcal{H}_{1}} u$ is spanned by $\left|i_{2}\right\rangle, i=1, \ldots, r$, and $\iota_{\mathcal{H}_{2}} \sigma(u)$ for the transposition $\sigma(f \otimes g)=g \otimes f$ is spanned by $\left|i_{1}\right\rangle, i=1, \ldots, r$. In other words the S-rank equals the Schmidt rank in this case, so the S-rank is a natural generalization of the latter.

\section{Entanglement and Segre maps for Bose and Fermi statistics}

Similarly to the case of distinguishable particles (see [8, 9]), the sets of all bosonic simple pure states (resp., fermionic simple pure states) can be described as the images of certain maps defined on the products of projective Hilbert spaces, the generalized Segre maps, as follows.

Consider first the standard Segre embedding $\mathrm{Seg}_{k}$ induced by the tensor product map: 


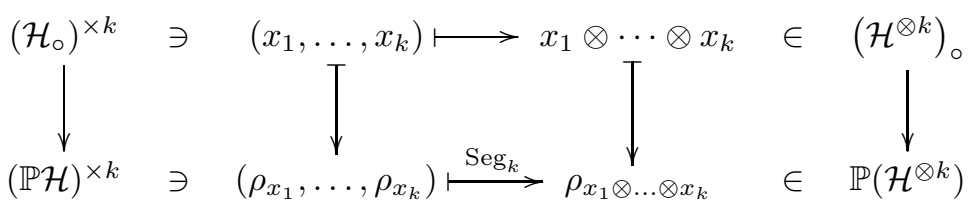

where $\mathcal{H}_{\circ}=\mathcal{H} \backslash\{0\}$.

It is clear that the analogous map, $\operatorname{Seg}_{k}^{\vee}$, for the Bose statistics should be

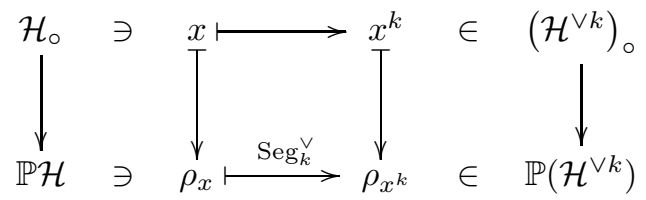

where $x^{k}=x \vee \cdots \vee x=x \otimes \cdots \otimes x$ ( $k$-factors), and for the Fermi statistics:

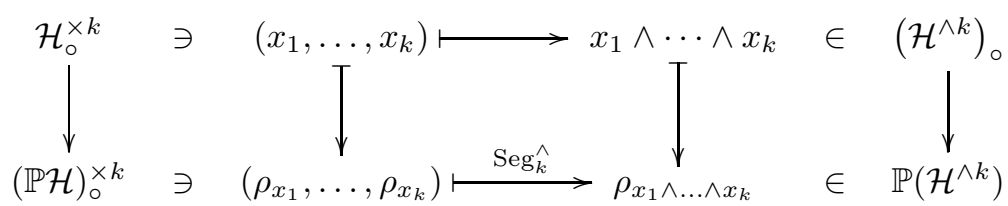

where $\mathcal{H}_{\circ}^{\times k}$ denotes

and $(\mathbb{P H})_{0}^{\times k}$ is

$$
\mathcal{H}^{\times k} \backslash\left\{\left(x_{1}, \ldots, x_{k}\right): x_{1} \wedge \cdots \wedge x_{k}=0\right\}
$$

$$
(\mathbb{P H})^{\times k} \backslash\left\{\left(\rho_{x_{1}}, \ldots, \rho_{x_{k}}\right): x_{1} \wedge \cdots \wedge x_{k}=0\right\} .
$$

Note that the condition $x_{1} \wedge \cdots \wedge x_{k} \neq 0$ does not depend on the choice of the vectors $x_{1}, \ldots, x_{k}$ in their projective classes and means that $\rho_{x_{1}}, \ldots, \rho_{x_{k}}$ do not lie in a common projective hyperspace. The subset $\mathcal{H}_{0}^{\times k}$ (resp., $(\mathbb{P H})_{0}^{\times k}$ ) is open and dense in $\mathcal{H}^{\times k}$ (resp., $\left.(\mathbb{P} \mathcal{H})^{\times k}\right)$. The following is an immediate consequence of Theorem 4.1 .

Theorem 5.1. A bosonic (fermionic) pure state $\rho \in \mathbb{P}\left(\mathcal{H}^{\vee k}\right)$ (resp., $\rho \in \mathbb{P}\left(\mathcal{H}^{\wedge k}\right)$ ) is entangled if and only if it lies outside the range of the Segre map

$$
\operatorname{Seg}_{k}^{\vee}: \mathbb{P H} \rightarrow \mathbb{P}\left(\mathcal{H}^{\vee k}\right) \quad\left(\text { resp. }, \quad \operatorname{Seg}_{k}^{\wedge}:(\mathbb{P H})_{\circ}^{\times k} \rightarrow \mathbb{P}\left(\mathcal{H}^{\wedge k}\right)\right) .
$$

A mixed bosonic (fermionic) state is entangled if and only if it lies outside the convex hull of the range of the corresponding Segre map.

\section{Entanglement for generalized parastatistics}

Our approach to the entanglement of composite systems for identical particles is so general and natural that it allows for an immediate implications also for generalized parastatistics. Parastatistics were introduced by Green 1 as a refinement of the spin-statistics connection introduced by Pauli 25. Green was motivated by a two-pages paper by Wigner addressing the connection between equations of motion and the commutation relations (Wigner's problem) [26, 27. The context of Green's paper is Quantum Field Theory, while most of the applications which have been proposed deal with thermodynamical aspects, in particular with the calculation of the partition function. In this paper we are concerned with parastatistics only to show that our proposed scheme applies to all the situations where states of composite systems are by construction not factorizable and are associated with representations of the permutation group acting on the tensor product of states of the subsystems. The reader who wants to know more about parastatistics could read [2, 28, 29.

Observe first that simple tensors of length 1 in $\tilde{\mathcal{H}}=\mathcal{H}_{1} \otimes \cdots \otimes \mathcal{H}_{k}$ form an orbit of the group $U\left(\mathcal{H}_{1}\right) \times$ $\cdots \times U\left(\mathcal{H}_{k}\right)$ acting on $\tilde{\mathcal{H}}$ in the obvious way. In fact, each such tensor can be written as $e_{1}^{1} \otimes \cdots \otimes e_{1}^{k}$ 
for certain choice of orthonormal bases $e_{1}^{j}, \ldots, e_{n_{j}}^{j}$ in $\mathcal{H}_{j}, j=1, \ldots, k$. This means that simple tensors are just vectors of highest (or lowest, depending on the convention) weight of the compact Lie group $U\left(\mathcal{H}_{1}\right) \times \cdots \times U\left(\mathcal{H}_{k}\right)$ relative to some choice of a maximal torus and Borel subgroups. If indistinguishable particles are concerned, the symmetric and antisymmetric tensors form particular irreducible parts of the 'diagonal' representation of the compact group $U(\mathcal{H})$ in the Hilbert space $\mathcal{H}^{\otimes k}$, defined by

$$
U\left(x_{1} \otimes \cdots \otimes x_{k}\right)=U\left(x_{1}\right) \otimes \cdots \otimes U\left(x_{k}\right)
$$

Recall that we identify the symmetry group $S_{k}$ with the group of certain unitary operators on the Hilbert space $\mathcal{H}^{k}$ in the obvious way,

$$
\sigma\left(x_{1} \otimes \cdots \otimes x_{k}\right)=x_{\sigma(1)} \otimes \cdots \otimes x_{\sigma(k)} .
$$

Note that the operators of $S_{k}$ intertwine the unitary action of $U(\mathcal{H})$. In the cases of the symmetric and antisymmetric tensors, we speak about the Bose and Fermi statistics, respectively. But, for $k>2$, there are other irreducible parts of the representation (39) associated with invariant subspaces of the $S_{k^{-}}$ action. We shall call them (generalized) parastatistics. Any of these $k$-parastatistics (i.e. any irreducible subspace of the tensor product $\mathcal{H}^{\otimes k}$ ) is associated with a Young tableau $\alpha$ with $k$-boxes (chambers) as follows (see e.g. 30, 31, 32]).

Consider partitions of $k: k=\lambda_{1}+\cdots+\lambda_{r}$, where $\lambda_{1} \geq \cdots \geq \lambda_{r} \geq 1$. To a partition $\lambda=\left(\lambda_{1}, \ldots, \lambda_{r}\right)$ is associated a Young diagram (sometimes called a Young frame or a Ferrers diagram) with $\lambda_{i}$ boxes in the $i$ th row, the rows of boxes lined up on the left. Define a tableau on a given Young diagram to be a numbering of the boxes by the integers $1, \ldots, k$, and denote with $Y_{\lambda}$ the set of all such Young tableaux. Finally, put $Y(k)$ to be the set of all Young tableaux with $k$ boxes. Given a tableau $\alpha \in Y(k)$ define two subgroups in the symmetry group $S_{k}$ :

$$
P=P_{\alpha}=\left\{\sigma \in S_{k}: \sigma \text { preserves each row of } \alpha\right\}
$$

and

$$
Q=Q_{\alpha}=\left\{\tau \in S_{k}: \tau \text { preserves each column of } \alpha\right\} .
$$

In the space of linear operators on $\mathcal{H}^{\otimes k}$ we introduce two operators associated with these subgroups:

$$
a_{\alpha}=\sum_{\tau \in Q}(-1)^{\tau} \tau, \quad b_{\alpha}=\sum_{\sigma \in P} \sigma .
$$

Finally, we define the Young symmetrizer

$$
c_{\alpha}=a_{\alpha} \circ b_{\alpha}=\sum_{\sigma \in P, \tau \in Q}(-1)^{\tau} \tau \circ \sigma .
$$

It is well known (see, e.g., 30, 31, 32] ) that $\pi^{\alpha}=\frac{1}{\mu(\alpha)} c_{\alpha}$, for some non-zero rational number $\mu(\alpha)$, is an orthogonal projector and that the image $\mathcal{H}^{\alpha}$ of $c_{\alpha}$ is an irreducible subrepresentation of $U(\mathcal{H})$, i.e. the parastatistics associated with $\alpha$. As a matter of fact, these representations for Young tableaux on the same Young diagram are equivalent, so that the constant $\mu(\alpha)$ depends only on the Young diagram $\lambda$ of $\alpha$ and does not depend on the enumeration of boxes. Hence, $\mu(\alpha)=\mu(\lambda)$ and this number is related to the multiplicity $m(\lambda)$ of the corresponding irreducible representation in $\mathcal{H}^{\otimes k}$ by $\mu(\lambda) \cdot m(\lambda)=k$ !. For a given Young diagram (partition) $\lambda$, the map

$$
\epsilon_{\lambda}=\frac{1}{\mu(\lambda)^{2}} \sum_{\alpha \in Y_{\lambda}} c_{\alpha}
$$

is an orthogonal projection, called the central Young symmetrizer, onto the invariant subspace being the sum of all copies of the irreducible representations equivalent to that with a parastatistics from $Y_{\lambda}$.

The symmetrization $\pi^{\vee}$ (antisymmetrization $\pi^{\wedge}$ ) projection corresponds to a Young tableau with just one row (one column) and arbitrary enumeration. It is well known that any irreducible representation $\mathcal{H}^{\alpha}$ of $U(\mathcal{H})$ contains cyclic vectors which are of highest weight relative to some choice of a maximal torus and Borel subgroups in $U(\mathcal{H})$. We will call them $\alpha$-simple tensors or simple tensors in $\mathcal{H}^{\alpha}$. Note 
that such vectors can be viewed as generalized coherent states [33. They can be also regarded as the 'most classical' states with respect to their correlation properties 34. These are exactly the tensors associated with simple (non-entangled) pure states for composite systems of particles with (generalized) parastatistics. This is because $\alpha$-simple tensors represent the minimal amount of quantum correlations for tensors in $\mathcal{H}^{\alpha}$, namely the quantum correlations forced directly by the particular parastatistics.

Example 6.1. (a) For $k=2$ we have just the obvious splitting of $\mathcal{H}^{\otimes 2}$ into symmetric and antisymmetric tensors: $\mathcal{H}^{\wedge 2} \oplus \mathcal{H}^{\vee 2}$.

(b) For $k=3$, besides symmetric and antisymmetric tensors associated with the Young tableaux

$$
\alpha_{0}=\begin{array}{|l|l|l|}
\hline 1 & 2 & 3 \\
\hline
\end{array} \text { and } \alpha_{3}=\begin{array}{|l|}
\hline 1 \\
\hline 2 \\
\hline 3 \\
\hline
\end{array}
$$

we have two additional irreducible parts associated with the Young tableaux

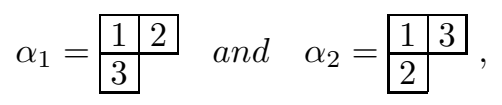

namely

$$
\mathcal{H}^{\otimes 3}=\mathcal{H}^{\wedge 3} \oplus \mathcal{H}^{\alpha_{1}} \oplus \mathcal{H}^{\alpha_{2}} \oplus \mathcal{H}^{\vee 3} .
$$

Since $P_{\alpha_{1}}=\{i d,(1,2)\}$ and $Q_{\alpha_{1}}=\{i d,(1,3)\}$, we get $a_{\alpha_{1}}=i d-(1,3)$ and $b_{\alpha_{1}}=i d+(1,2)$, so that

$$
c_{\alpha_{1}}=a_{\alpha_{1}} \circ b_{\alpha_{1}}=(i d-(1,3)) \circ(i d+(1,2))=i d+(1,2)-(1,3)-(123) .
$$

As the multiplicity of the representation is 2 , we have $\mu\left(\alpha_{1}\right)=3 ! / 2=3$ and the projection

$$
\pi^{\alpha_{1}}: \mathcal{H}^{\otimes 3} \rightarrow \mathcal{H}^{\alpha_{1}}
$$

takes the form

$$
\pi^{\alpha_{1}}\left(x_{1} \otimes x_{2} \otimes x_{3}\right)=\frac{1}{3}\left(x_{1} \otimes x_{2} \otimes x_{3}+x_{2} \otimes x_{1} \otimes x_{3}-x_{3} \otimes x_{2} \otimes x_{1}-x_{3} \otimes x_{1} \otimes x_{2}\right) .
$$

Similarly,

$$
\begin{gathered}
\pi^{\alpha_{2}}: \mathcal{H}^{\otimes 3} \rightarrow \mathcal{H}^{\alpha_{2}} \\
\pi^{\alpha_{2}}\left(x_{1} \otimes x_{2} \otimes x_{3}\right)=\frac{1}{3}\left(x_{1} \otimes x_{2} \otimes x_{3}+x_{3} \otimes x_{2} \otimes x_{1}-x_{2} \otimes x_{1} \otimes x_{3}-x_{2} \otimes x_{3} \otimes x_{1}\right) .
\end{gathered}
$$

The simple tensors (the highest weight vectors) in $\mathcal{H}^{\alpha_{1}}$ can be written as

$$
v_{\lambda}^{\alpha_{1}}=\lambda\left(e_{1} \otimes e_{1} \otimes e_{2}-e_{2} \otimes e_{1} \otimes e_{1}\right),
$$

for certain choice of an orthonormal basis $e_{i}$ in $\mathcal{H}$ and $\lambda \neq 0$. Analogously, the simple tensors in $\mathcal{H}^{\alpha_{2}}$, in turn, take the form

$$
v_{\lambda}^{\alpha_{2}}=\lambda\left(e_{1} \otimes e_{2} \otimes e_{1}-e_{2} \otimes e_{1} \otimes e_{1}\right) .
$$

For $\operatorname{dim}(\mathcal{H})=3$, the simple tensors of length 1 form an orbit of the unitary group $U(\mathcal{H})$ of the (real) dimension 7 in $\mathcal{H}^{\alpha_{1}}$ and $\mathcal{H}^{\alpha_{2}}$. The simple symmetric tensors of length 1 form an orbit of the dimension 5 , and the simple antisymmetric ones (of length 1 ) - an orbit of the dimension 1 . The dimensions of the irreducible representations are: $\operatorname{dim}\left(\mathcal{H}^{\wedge 3}\right)=1, \operatorname{dim}\left(\mathcal{H}^{\vee 3}\right)=10, \operatorname{dim}\left(\mathcal{H}^{\alpha_{1}}\right)=\operatorname{dim}\left(\mathcal{H}^{\alpha_{2}}\right)=8$.

A fundamental observation is that $\alpha$-simple tensors can also be characterized in terms of the S-rank.

Theorem 6.1. A tensor $v \in \mathcal{H}^{\alpha}, \alpha \in Y(k)$, is simple if and only if it has the minimal S-rank among non-zero tensors from $\mathcal{H}^{\alpha}$. This minimal S-rank equals the number $r$ of rows in the corresponding Young diagram and the simple tensor reads as

$$
v=\pi^{\alpha}\left(e_{\alpha(1)} \otimes \cdots \otimes e_{\alpha(k)}\right)
$$


where $e_{1}, \ldots, e_{r}$ are some linearly independent vectors in $\mathcal{H}$ and $\alpha(i)$ is the number of the row in which the box with the number $i$ appears in the tableaux $\alpha$. In other words, the tensor

$$
E_{\alpha}=e_{\alpha(1)} \otimes \cdots \otimes e_{\alpha(k)}
$$

is the tensor product of $k$ vectors from the sequence $E=\left(e_{1}, \ldots, e_{r}\right)$ obtained by putting $e_{j}$ in the places indicated by the number of the boxes in the jth row.

Proof. Assume that $v$ is of the form (51). Passing to the complexification $G L(\mathcal{H})$ of $U(\mathcal{H})$ and using a basis $e_{1}, \ldots, e_{n}$ extending the linear independent family $e_{1}, \ldots, e_{r} \in \mathcal{H}$ to identify $G L(\mathcal{H})$ with $G L(n ; \mathbb{C})$, we easily see that $E_{\alpha}$, thus $v$, is an eigenvector for any diagonal matrix and is killed by any uppertriangular matrix. Moreover, $v \neq 0$, hence $v$ is a vector of highest weight. Indeed, by definition $b_{\alpha}\left(E_{\alpha}\right)$ is non-zero and proportional to $E_{\alpha}$, so that $v=t \cdot a_{\alpha}\left(E_{\alpha}\right)$ for a non-zero constant $t$. It is now easy to see that $\left\{\tau\left(E_{\alpha}\right): \tau \in Q\right\}$ is a family of linearly independent tensors in $\mathcal{H}^{\otimes k}$, so that $v=\sum_{\tau \in Q}(-1)^{\tau} \tau\left(E_{\alpha}\right)$ is non-zero. As tensors from $\left\{\tau\left(E_{\alpha}\right): \tau \in Q\right\}$ are linearly independent and have one of $e_{1}, \ldots, e_{r}$ as the last factor, the S-rank of $v$ is at least $r$. On the other hand, $v$ is composed from tensor products of $e_{1}, \ldots, e_{r}$ only, so the S-rank is at most $r$.

Conversely, let $v \in \mathcal{H}^{\alpha}, v \neq 0$. Without loss of generality we can assume that the numbers in the first column of the Young tableaux $\alpha$ are $k-r+1, \ldots, k$. Since $\epsilon_{\lambda}(v)=v$, we have

$$
v=\frac{1}{\mu(\lambda)^{2}} \sum_{\varsigma \in S_{r}}(-1)^{\varsigma} \varsigma\left(\sum_{\tau \in Q^{\prime}}(-1)^{\tau} \tau\left(b_{\alpha}(v)\right)\right),
$$

where

$$
Q^{\prime}=\{\tau \in Q: \tau \text { is identical on the first column of } \alpha\}
$$

and $S_{r}$ is the permutation group of $\{k-r+1, \ldots, k\}$. This means that $v$ is skew-symmetric with respect to the last $r$ positions, so the contractions $i_{\nu} v$, with $\nu \in(\mathcal{H})^{\otimes(k-1)}$, can be written as contractions of a skew-symmetric $r$-tensor, thus they span a vector space of dimension $\geq r$ (see Theorem 4.1 (c)). If this dimension is exactly $r$, then $v$ can be written as a combination of linearly independent tensor products $e_{s(1)} \otimes \cdots \otimes e_{s(k)}$ of vectors from $\left\{e_{1}, \ldots, e_{r}\right\}$. Since the tensor is symmetric with respect to permutations preserving rows and skew-symmetric with respect to permutations of the first column, each tensor product $e_{s(1)} \otimes \cdots \otimes e_{s(k)}$ in this combination should satisfy $e_{s(i)}=e_{s_{(j)}}$ if $i$ and $j$ are in the same row of $\alpha$. Hence, $v$ is proportional to $\pi^{\alpha}\left(E_{\alpha}\right)$.

Let $\mathcal{H}^{\alpha} \subset \mathcal{H}^{\otimes k}$ denotes the irreducible component of the tensor representation of the unitary group $U(\mathcal{H})$ in $\mathcal{H}^{\otimes k}$ associated with a Young diagram $\alpha \in Y(k)$.

\section{Definition 6.1.}

(a) We say that a pure state $\rho_{v}$ on $\mathcal{H}^{\otimes k}$ obeys a parastatistics $\alpha \in Y(k)$ (is a pure $\alpha$-state for short) if $v \in \mathcal{H}^{\alpha}$, i.e. $\rho$ is a pure state on the Hilbert space $\mathcal{H}^{\alpha}$.

(b) A pure state $\rho$ on $\mathcal{H}^{\otimes k}$ obeying a parastatistics $\alpha$ is called a simple pure state for the parastatistics $\alpha$ (simple pure $\alpha$-state for short) if $\rho$ is represented by an $\alpha$-simple tensor in $\mathcal{H}^{\alpha}$. If $\rho$ is not simple $\alpha$-state, we call it an entangled pure $\alpha$-state.

(c) A mixed state $\rho$ on $\mathcal{H}^{\alpha}$ we call a simple (mixed) state for the parastatistics $\alpha$ (simple $\alpha$-state for short), if it can be written as a convex combination of simple pure $\alpha$-states. In the other case, $\rho$ is called an entangled mixed $\alpha$-state.

\section{$7 \quad$ Segre maps for generalized parastatistics}

In general, for an arbitrary parastatistics (Young tableau) $\alpha \in Y(k)$ with the partition (Young diagram) $\lambda=\left(\lambda_{1}, \ldots, \lambda_{r}\right)$, we define the generalized Segre map $\operatorname{Seg}^{\alpha}(\alpha$-Segre map $)$ as a map $\operatorname{Seg}^{\alpha}:(\mathbb{P H})_{0}^{\times r} \rightarrow$ $\mathbb{P}\left(\mathcal{H}^{\alpha}\right)$ described as follows. 
Let us consider first the map

$$
i_{\alpha}: \mathcal{H}^{\times r} \rightarrow \mathcal{H}^{\otimes k}, \quad\left(x_{1}, \ldots, x_{r}\right) \mapsto x_{\alpha(1)} \otimes \cdots \otimes x_{\alpha(k)},
$$

where $\alpha(i)$ is the number of the row in which the box with the number $i$ appears in the tableaux $\alpha$. In other words, we make a tensor product of $k$ vectors from $\left\{x_{1}, \ldots, x_{r}\right\}$ by putting $x_{j}$ in the places indicated by the number of the boxes in the $j$ th row. For instance, the Young tableaux from Example 6.1 give $i_{\alpha_{1}}\left(x_{1}, x_{2}\right)=x_{1} \otimes x_{1} \otimes x_{2}$ and $i_{\alpha_{2}}\left(x_{1}, x_{2}\right)=x_{1} \otimes x_{2} \otimes x_{1}$. It is clear that $i_{\alpha}\left(x_{1}, \ldots, x_{r}\right)$ is an eigenvector of $b_{\alpha}$.

The Segre map $\operatorname{Seg}^{\alpha}$ associates with $\left(\rho_{x_{1}}, \ldots, \rho_{x_{r}}\right) \in(\mathbb{P} \mathcal{H})_{\circ}^{\times r}$ the pure state $\rho_{\pi^{\alpha}\left(x_{\alpha(1)} \otimes \cdots \otimes x_{\alpha(k)}\right)}$ in $\mathcal{H}^{\alpha}$, as shows the following diagram:

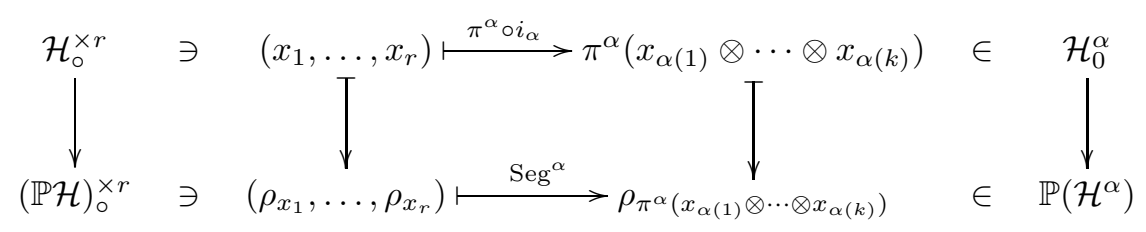

Note that $\pi^{\alpha}\left(x_{\alpha(1)} \otimes \cdots \otimes x_{\alpha(k)}\right)$ is proportional to the antisymmetrization of the tensor $x_{\alpha(1)} \otimes \cdots \otimes x_{\alpha(k)}$ and that the construction is correct, since $\pi^{\alpha}\left(x_{\alpha(1)} \otimes \cdots \otimes x_{\alpha(k)}\right)$ is non-zero if and only if $x_{1} \wedge \cdots \wedge x_{r} \neq 0$, and its projective class is uniquely determined by the projective classes of $x_{1}, \ldots, x_{r}$. Note also that we can always take $x_{1}, \ldots, x_{r}$ orthogonal, say, $x_{1}=e_{1}, \ldots, x_{r}=e_{r}$, since the antisymmetrization kills the part of $x_{i}$ which is the orthogonal projection of $x_{i}$ onto the linear subspace spanned by the rest of the vectors $x_{j}$. Now, according to Theorem 6.1, $\pi^{\alpha}\left(x_{\alpha(1)} \otimes \cdots \otimes x_{\alpha(k)}\right)$ is $\alpha$-simple, so that $\rho_{\pi^{\alpha}\left(x_{\alpha(1)} \otimes \cdots \otimes x_{\alpha(k)}\right)}$ is a simple pure $\alpha$-state. Moreover, each simple pure $\alpha$-state is of this form and for symmetric and antisymmetric tensors this construction agrees with (37) and (38). We therefore get the following.

Theorem 7.1. A pure $\alpha$-state $\rho \in \mathbb{P}\left(\mathcal{H}^{\alpha}\right)$ is an entangled $\alpha$-state if and only if it is not in the range of the Segre map

$$
\operatorname{Seg}^{\alpha}:(\mathbb{P H})_{\circ}^{\times r} \rightarrow \mathbb{P}\left(\mathcal{H}^{\alpha}\right)
$$

The set $\mathcal{S}\left(\mathcal{H}^{\alpha}\right)$ of mixed non-entangled $\alpha$-states is the convex hull of the range of the normalized $\alpha$-Segre map,

$$
\mathcal{S}\left(\mathcal{H}^{\alpha}\right)=\operatorname{conv}\left(\operatorname{Seg}^{\alpha}\left(\mathcal{P}(\mathcal{H})_{\alpha}^{\times r}\right)\right)
$$

and mixed entangled $\alpha$-states are exactly members of

$$
\mathcal{D}\left(\mathcal{H}^{\alpha}\right) \backslash \mathcal{S}\left(\mathcal{H}^{\alpha}\right)
$$

Let us observe that

$$
\left\|\pi^{\alpha}\left(x_{\alpha(1)} \otimes \cdots \otimes x_{\alpha(k)}\right)\right\|^{2} \cdot \rho_{\pi^{\alpha}\left(x_{\alpha(1)} \otimes \cdots \otimes x_{\alpha(k)}\right)}=\pi^{\alpha} \circ\left(\rho_{x_{\alpha(1)}} \otimes \cdots \otimes \rho_{x_{\alpha(k)}}\right) \circ \pi^{\alpha},
$$

as operators on $\mathcal{H}^{\otimes k}$. Indeed, since $\pi^{\alpha}$ is an orthogonal projection, the left-hand side equals

$$
\begin{gathered}
\left|\pi^{\alpha}\left(x_{\alpha(1)} \otimes \cdots \otimes x_{\alpha(k)}\right)\right\rangle\left\langle\pi^{\alpha}\left(x_{\alpha(1)} \otimes \cdots \otimes x_{\alpha(k)}\right)\right|(y)= \\
\pi^{\alpha}\left(x_{\alpha(1)} \otimes \cdots \otimes x_{\alpha(k)}\right)\left\langle x_{\alpha(1)} \otimes \cdots \otimes x_{\alpha(k)} \mid \pi_{\alpha}(y)\right\rangle=\left(\pi^{\alpha} \circ\left(\rho_{x_{\alpha(1)}} \otimes \cdots \otimes \rho_{x_{\alpha(k)}}\right) \circ \pi^{\alpha}\right)(y) .
\end{gathered}
$$

This suggests to look at the map (the big $\alpha$-Segre map):

$$
\widetilde{\operatorname{Seg}^{\alpha}}:\left(\mathfrak{u}^{*}(\mathcal{H})\right)^{\times r} \rightarrow \mathfrak{u}^{*}\left(\mathcal{H}^{\alpha}\right) \subset \mathfrak{u}^{*}\left(\mathcal{H}^{\otimes k}\right),
$$

where $\mathfrak{u}^{*}(\mathcal{H})$ denotes the (real) vector space of selfadjoint operators on $\mathcal{H}$, defined by

$$
\widetilde{\operatorname{Seg}^{\alpha}}\left(u_{1}, \ldots, u_{r}\right)=\pi_{\alpha} \circ\left(u_{\alpha(1)} \otimes \cdots \otimes u_{\alpha(k)}\right) \circ \pi_{\alpha}
$$

The big $\alpha$-Segre map is a natural generalization of the map (7). A closer study of the big Segre maps we postpone to a separate paper. 


\section{Conclusions}

The presented geometric description, in terms of Segre maps, of entanglement properties for systems with arbitrary statistics parallels our previous algebraic approach to such systems based on the concept of Srank of a tensor. It puts on equal and unifying footing systems of distinguishable particles, for which both algebraic and geometric descriptions were known, and systems with indistinguishable particles. What is more, this description provides an explicit form of simple (separable) pure states for an arbitrary parastatistics an effective procedures to check the simplicity in the bosonic and the fermionic case. Such procedures for arbitrary parastatistics are not known for us and can be the subject of forthcoming papers. Also the problem of the decomposition of the total algebra of operators, associated with the S-rank, is an interesting and open question.

\section{Acknowledgments}

Research of the first two authors was financed by the Polish Ministry of Science and Higher Education under the grant No. N N202 090239. G. Marmo would like to acknowledge the support provided by the Santander/UCIIIM chair of Excellence programme 2011-2012. We are also indebted to referees for their useful comments and suggestions.

\section{References}

[1] H. S. Green. A generalized method of field quantization. Phys. Rev., 90:270?273, 1953.

[2] Y. Ohnuki and S. Kamefuchi. Quantum Field Theory and Parastatistics. Springer, Berlin (1982).

[3] J. Grabowski, M. Kuś, and G. Marmo. Entanglement for multipartite systems of indistinguishable particles. J. Phys. A: Math. Gen. 44:175302 (21pp), 2011.

[4] J. Schliemann, D. Loss, and A. H. MacDonald. Double-occupancy errors, adiabaticity, and entanglement of spin qubits in quantum dots. Phys. Rev. B, 63(8):085311, 2001.

[5] J. Schliemann, J. I. Cirac, M. Kuś, M. Lewenstein, and D. Loss. Quantum correlations in twofermion systems. Phys. Rev. A, 64:022303, 2001.

[6] K. Eckert, J. Schliemann, D. Bruß, and M Lewenstein. Quantum correlations in systems of identical particles. Ann. Phys., 299:88-127, 2002.

[7] R. Paškauskas and L. You. Quantum correlations in two-boson wave functions. Phys. Rev. A, 64(4):042310, 2001.

[8] J. Grabowski, M. Kuś, and G. Marmo. Geometry of quantum systems: density states and entanglement. J. Phys. A: Math. Gen. 38:10217-10244, 2005.

[9] J. Grabowski, M. Kuś, and G. Marmo. Symmetries, group actions, and entanglement. Open Sys. Information Dyn., 13:343-362, 2006.

[10] Y. S. Li, B. Zeng, X. S. Liu, and G. L. Long. Entanglement in a two-identical-particle system. Phys. Rev. A, 64(5):054302, 2001.

[11] G. Ghirardi, L. Marinatto, and T. Weber. Entanglement and Properties of Composite Quantum Systems: A Conceptual and Mathematical Analysis. Journal of Statistical Physics, 108(1):49-122, 2002.

[12] G. Ghirardi and L. Marinatto. General criterion for the entanglement of two indistinguishable particles. Phys. Rev. A, 70(1):012109, 2004.

[13] G. Ghirardi and L. Marinatto. Identical particles and entanglement. Optics and Spectroscopy, 99(3):386-390, 2005. 
[14] F. Buscemi, P. Bordone, and A. Bertoni. Linear entropy as an entanglement measure in two-fermion systems. Phys. Rev. A 75:032301, 2007.

[15] A. R. Plastino, D. Manzano, and J. S. Dehesa. Separability Criteria and Entanglement Measures for Pure States of N Identical Fermions. Europhys. Lett. 86:20005, 2009.

[16] B. Lari, P. Durganandini, and P. S. Joag. Multipartite entanglement in fermionic systems via a geometric measure. Phys. Rev. A 82:062302, 2010.

[17] F. Buscemi and P. Bordone A measure of tripartite entanglement in bosonic and fermionic systems. Phys. Rev. A 84:022303, 2011.

[18] P. Zanardi. Quantum entanglement in fermionic lattices. Phys. Rev. A 65:042101(R), 2002.

[19] P. Zanardi and and X. Wang Fermionic entanglement in itinerant systems. J. Phys. A 35:7947-7959, 2002.

[20] M. R. Dowling, A. C. Doherty, and H. M. Wiseman. Entanglement of indistinguishable particles in condensed matter physics Phys. Rev. A 73:052323, 2006.

[21] T. Sasaki, T. Ichikawa, and I. Tsutsui. Entanglement of indistinguishable particles. Phys. Rev. A 83:012113, 2011.

[22] M. C. Tichy, F. Mintert, and A. Buchleitner Essential entanglement for atomic and molecular physics. J. Phys. B: At. Mol. Opt. Phys. 44:192001, 2011.

[23] G. Vidal. Entanglement monotones. J. Mod. Opt. 47:355-376, 2000.

[24] R. Hartshorne. Algebraic geometry. Springer, 1977, Sect. IV.2.

[25] W. Pauli. On the connection between spin and statistics Phys. Rev. 58:716-728, 1940.

[26] E. P. Wigner. Do the equations of motion determine the quantum mechanical commutation relations? Phys. Rev. 77:711-712, 1950.

[27] V. I. Man'ko, G. Marmo, E. C. G. Sudarshan, and F. Zaccaria. Wigner's Problem and Alternative Commutation Relations for Quantum Mechanics Int. J. Mod. Phys. B 11:1281-1296, 1997.

[28] A. M. L. Messiah, O. W. Greenberg. Symmetrization postulate and its experimental foundation. Phys. Rev.(2) 136:B248-B267, 1964.

[29] R. Y. Cusson. Examples of parastatistics Ann. Physics 55:22-40, 1969.

[30] W. Fulton and J. Harris. Representation Theory. A First Course. Springer Verlag, 1991.

[31] W. Fulton Young Tableaux. Cambridge University Press, 1997.

[32] D. P. Zhelobenko. Compact Lie Groups and Their Representations. Amer. Math. Soc., 1973 (Translated from Russian).

[33] A. Perelomov. Generalized coherent states and their applications. Springer, Heidelberg, 1986.

[34] M. Kuś and I. Bengtsson. 'Classical' quantum states. Phys. Rev. A 80:022319, 2009. 